\title{
Development of Large Crystal Size Nuclear Emulsion for Cosmic-ray Radiography
}

\author{
Akira NISHIO ${ }^{1}$ \\ Graduate School of Science, Nagoya University \\ Fro-cho, Chikusa-ku, Nagoya, 464-8602, Japan \\ E-mail: nishiodflab.phys.nagoya-u.ac.jp
}

\section{Masaki MOTO}

Graduate School of Science, Nagoya University

Fro-cho, Chikusa-ku, Nagoya, 464-8602, Japan

E-mail: motolflab.phys.nagoya-u.ac.jp

\section{Yuta MANABE}

Graduate School of Science, Nagoya University

Fro-cho, Chikusa-ku, Nagoya, 464-8602, Japan

E-mail: manabedflab.phys.nagoya-u.ac.jp

\section{Ken-ichi KUWABARA}

Graduate School of Science, Nagoya University

Fro-cho, Chikusa-ku, Nagoya, 464-8602, Japan

E-mail: kenichi-kuwabara@nifty.com

\section{Kunihiro MORISHIMA}

Institute For Advanced Research, Nagoya University

Fro-cho, Chikusa-ku, Nagoya, 464-8602, Japan

E-mail: morishima@flab.phys.nagoya-u.ac.jp

Nuclear emulsion is a kind of photographic film and has sensitivity for ionizing radiation. The film record tracks of charged particle with angular accuracy under several mrad. We are developing nuclear emulsion with crystal size larger than conventional $200 \mathrm{~nm}$ one. Large crystal size nuclear emulsion has a potential of high sensitivity and contrast, so it is hopeful as future cosmic-ray radiography detector. We succeeded in producing emulsion gels which have silver bromide crystal with diameter 200, 350, 450 and $800 \mathrm{~nm}$ using emulsion gel manufacturing equipment in Nagoya University. After applying optimum sensitization and development processing to each crystal size, sensitivity (G.D) and noise (F.D) ware as follows.

$$
\begin{array}{ll}
200 \mathrm{~nm}: \mathrm{G} . \mathrm{D}=33.6 \pm 2.6 & \mathrm{FD}=1.1 \pm 0.2 \\
350 \mathrm{~nm}: \mathrm{G} . \mathrm{D}=42.0 \pm 2.9 & \mathrm{FD}=0.9 \pm 0.2 \\
450 \mathrm{~nm}: \mathrm{G} . \mathrm{D}=46.6 \pm 3.1 & \mathrm{FD}=0.5 \pm 0.1 \\
800 \mathrm{~nm}: \mathrm{G} . \mathrm{D}=(34.1 \pm 3.8) & \mathrm{FD}=0.7 \pm 0.2
\end{array}
$$

The 3rd International Symposium on "Quest for the Origin of Particles and the Universe" 5-7 January 2017

Nagoya University, Japan

\section{${ }^{1}$ Speaker}




\section{Introduction}

Cosmic ray radiography is the a new non-destructive inspection technique of large-scale constructions with cosmic ray muon. Cosmic ray muon has high penetrating power and it always comes from the whole sky. In the same way of taking a X-ray photograph, we can obtain integrated density of constructions which thickness are several tens to several hundreds. We had ever applied this technique to pyramids, nuclear reactors, volcanos, and so on [1] [2]. In these observations, we used nuclear emulsion as a detector. Nuclear emulsion is a kind of photographic film and has sensitivity for ionizing radiation. The film record tracks of charged particle with angular accuracy under several mrad. Nuclear emulsion is made by coating emulsion gel on plastic base. We put the emulsion gel production machine into operation in 2010 at Nagoya University. It was enable us to develop new-type emulsion gel by ourself. Emulsion gel is mainly consisted by silver bromide crystal and gelatin. We report about properties of Large Crystal Size Nuclear Emulsion developed in Nagoya University.

\section{Large Crystal Size Nuclear Emulsion}

We developed a Large Crystal Nuclear Emulsion for the purpose of improving sensitivity, contrast, and long-term characteristics. Emulsion gel manufacturing equipment in Nagoya University was used to produce the new-type emulsion gel. We have succeeded in producing nuclear emulsion with the diameter of silver bromide crystals at $350,450,800 \mathrm{~nm}$, in addition to the conventional $200 \mathrm{~nm}$ one. Electron microscopic image of the silver bromide crystal are shown in Figure 1.
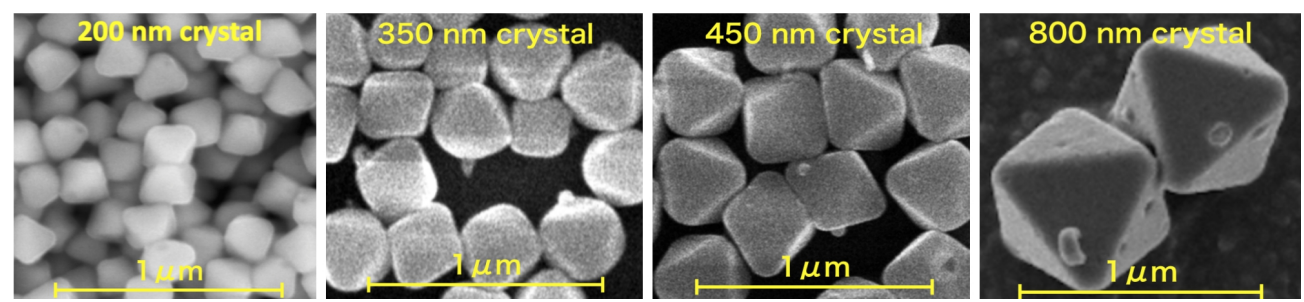

Fig. 1 Electron microscopic image of the conventional $200 \mathrm{~nm}$ silver bromide crystal and new-type 350, 450, $800 \mathrm{~nm}$ crystal (SEM image).

\section{Sensitivity and Noise}

Optimum gold-sulfur sensitization was performed to each crystal size [3]. For the developing solution, XAA developer (FUJI FILM Co. Ltd.) was used. pH of the developing solution was optimally adjusted to each crystal size. Figure 2 shows the optical microscopic image of minimum ionized particle tracks. Table 1 shows the evaluation results of sensitivity (Grain Density) and noise (Fog Density). The volume occupancy of silver bromide was $30 \%$.
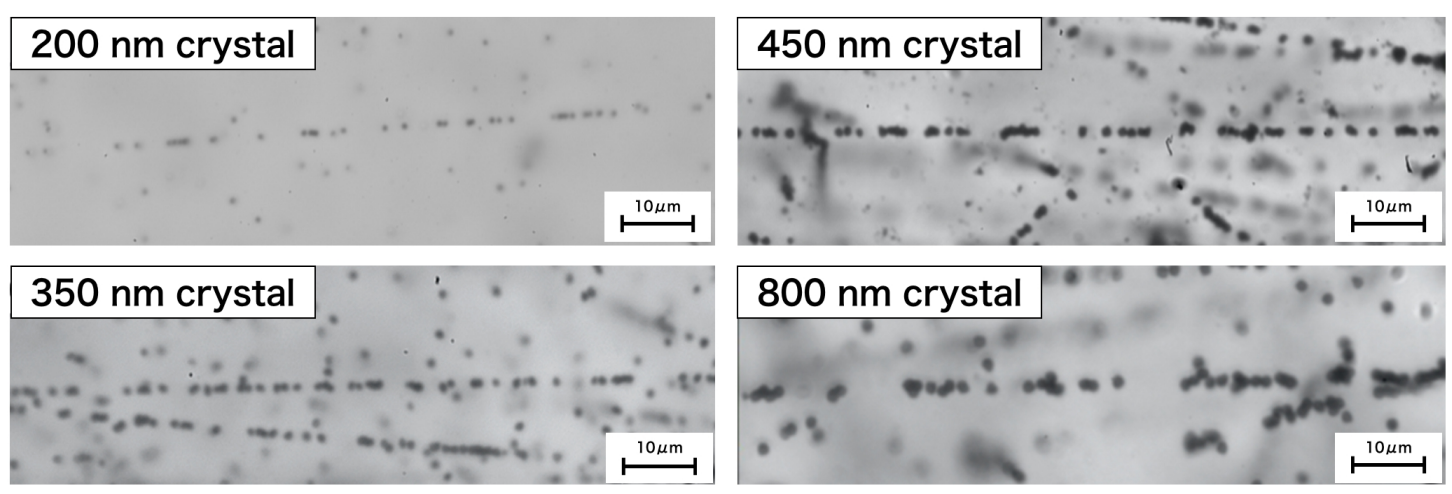

Fig.2 Optical microscopic image of minimum ionized particle tracks. The larger the silver bromide crystals, the higher the contrast. 


\begin{tabular}{l|rrrr} 
Crystal Size $(\mathrm{nm})$ & 200 & 350 & 450 & 800 \\
\hline \hline Grain Density $(/ 100 \mu \mathrm{m})$ & $33.6 \pm 2.6$ & $42.0 \pm 2.9$ & $46.6 \pm 3.1$ & $(34.1 \pm 3.8)$ \\
\hline Number of Crystal $(/ 100 \mu \mathrm{m})$ & 225 & 129 & 100 & 56 \\
\hline Crystal Sensitivity & $0.15 \pm 0.01$ & $0.33 \pm 0.02$ & $0.47 \pm 0.03$ & $(0.61 \pm 0.07)$ \\
\hline \hline Fog Density $\left(/ 1000 \mu \mathrm{m}^{3}\right)$ & $1.1 \pm 0.2$ & $0.9 \pm 0.2$ & $0.5 \pm 0.1$ & $0.7 \pm 0.2$
\end{tabular}

Table.1 Sensitivity and noise of Large Crystal Size Nuclear Emulsion. Sensitivity is evaluated by Grain Density (G.D) which is linear density of developed crystals. Noise is evaluated by Fog Density (F.D) which is volume density of randomly developed crystals. Grain Density of $800 \mathrm{~nm}$ crystals was shown with parenthesis due to reproducibility problem.

\section{Conclusion}

We developed nuclear emulsion with crystal size larger than conventional $200 \mathrm{~nm}$ one. We established producing method of emulsion gels which have silver bromide crystal with diameter 200, 350, 450 and $800 \mathrm{~nm}$ using emulsion gel manufacturing equipment in Nagoya University. By applying optimum chemical sensitization and development for each crystal size, we succeeded in increasing the sensitivity without changing the noise level compared to the conventional $200 \mathrm{~nm}$ one. Furthermore, as contrast improved as the crystal size was larger, it is suitable for future low magnification / wide field scanning.

\section{References}

[1] Hiroyuki KM Tanaka, Toshiyuki Nakano, Satoru Takahashi, Jyunya Yoshida, Minoru Takeo, Jun Oikawa, Takao Ohminato, Yosuke Aoki, Etsuro Koyama, Hiroshi Tsuji, et al. High resolution imaging in the inhomogeneous crust with cosmic-ray muon radiography: The density structure below the volcanic crater floor of mt. asama, japan. Earth and Planetary Science Letters, Vol. 263, No. 1, pp. 104-113, 2007.

[2] Kunihiro Morishima, Naotaka Naganawa, Toshiyuki Nakano, and Mitsuhiro Nakamura. First demonstration of cosmic ray muon radiography of reactor cores with nuclear emulsions based on an automated high-speed scanning technology. RADIATION DETECTORS AND THEIR USES, $p$. 30, 2012.

[3] M. Moto et al., 2nd Fall meeting of Federation of Imaging Societies. pp. 29-30 (2015) . 